\title{
Saccadic Eye Movements are Related to Turning Performance in Parkinson Disease
}

\author{
Corey A. Lohnes ${ }^{\mathrm{a}}$ and Gammon M. Earhart ${ }^{\mathrm{a}, \mathrm{b}, \mathrm{c}, *}$ \\ a Program in Physical Therapy, Washington University in St. Louis, St. Louis, MO, USA \\ ${ }^{\mathrm{b}}$ Department of Anatomy \& Neurobiology, Washington University in St. Louis, St. Louis, MO, USA \\ ${ }^{\mathrm{c}}$ Department of Neurology, Washington University in St. Louis, St. Louis, MO, USA
}

\begin{abstract}
Background. Persons with Parkinson disease (PD) experience difficulty turning, leading to freezing of gait and falls. We hypothesized that saccade dysfunction may relate to turning impairments, as turns are normally initiated with a saccade.

Objective. Determine whether saccades are impaired during turns in PD and if characteristics of the turn-initiating saccade are predictive of ensuing turn performance.

Methods. 23 persons with PD off medication and 19 controls performed 90 and 180 degree in-place turns to the right and left. Body segment rotations were measured using 3-D motion capture and oculomotor data were captured using a head-mounted eye tracking system and electrooculography. Total number of saccades and the amplitude, velocity, and timing of the first saccade were determined.

Results. Turn performance (turn duration, number of steps to turn) was impaired in PD $(p<0.05)$. PD performed more saccades, and the velocity and timing of the first saccade was impaired for both turn amplitudes $(p<0.05)$. Amplitude of the first saccade was decreased in PD during 180 degree turns. Turn duration correlated with oculomotor function. Characteristics of the first saccade explained $48 \%$ and $58 \%$ of the variance in turn duration for 90 and 180 degree turns, respectively.

Conclusions. Turning performance is impaired in PD and may be influenced by saccade dysfunction. An association between saccade function and turning performance may be indicative of the key role of saccades in initiating proper turning kinematics. Future work should focus on improving saccade performance during functional tasks and testing the effects of therapeutic interventions on related outcomes.
\end{abstract}

Keywords: Parkinson disease, saccades, oculomotor dysfunction, gait, turning

\section{INTRODUCTION}

Parkinson disease (PD) is a progressive neurodegenerative disease that is associated with a reduction in mobility, with problems that include difficulty turning. Turning difficulties can lead to freezing of gait (FOG), falls, fear of falling, and social withdrawal [1-3]. Falls that occur during turning are eight times more likely to result in hip fracture than falls during straight line

\footnotetext{
*Correspondence to: Gammon M. Earhart, PT, PhD, Program in Physical Therapy, Washington University School of Medicine, Campus Box 8502, 4444 Forest Park Blvd., St. Louis, MO, 63108. Tel.: 314286 1425; Fax: 314286 1410; E-mail: earhartg@wusm. wustl.edu.
}

walking [4]. Furthermore, individuals with PD have a 3.2 fold greater risk of hip fracture than age-matched individuals without PD [5]. In addition to the large personal cost of turning difficulties, hip fractures represent a substantial financial burden to society, with the cost of hip fracture care in individuals with PD totaling approximately $\$ 192$ million per year in the United States [5, 6].

Studies focusing on turning have noted that individuals with $\mathrm{PD}$ require more steps and take longer to complete a turn than healthy controls [7-11]. Those with PD who report turning difficulty also have a higher incidence of freezing of gait and falls $[10,12]$. Furthermore, the timing of segmental rotations during turn initiation is altered in PD. This has been termed "en 
bloc" turning and is characterized by the near simultaneous rotation of the head, trunk, and pelvis and reduced relative rotations between adjacent segments $[9,13-15]$. Other measures of poor turn quality have been observed in those with PD including a wider turn arc [16], narrowed step width [11, 16, 17], and higher variation in step duration compared with controls [16].

It is evident that visual information plays an important role in the control of locomotion and turning. Clear differences in gaze behavior and stepping performance have been demonstrated between older adult fallers and non-fallers [18]. In addition, training of eye movements has been shown to improve locomotor performance in individuals with cerebellar damage [19]. Several studies in healthy individuals have shown that the eyes participate in a top-down rotation sequence such that the eyes are the first to turn, followed by the head, trunk, and then the feet. The initial saccade during a turn, in combination with subsequent head movements, provides a shift of gaze to a position aligned with the direction of travel. Gaze shifts precede shifts in center of mass (COM) trajectory during turning and unexpected perturbations of gaze cause delays in COM movement to steer the body along the desired trajectory [24].

While eye movements have been measured in healthy adults during turning tasks, it is unclear how eye movements relate to turning performance in individuals with PD. During head-fixed tasks, saccadic eye movements have been shown to be abnormal in those with PD, including prolonged fixation times, bradykinesia, and akinesia during rapid alternating gaze shifts between two fixed targets. Several more recent studies have demonstrated deficits in control of voluntary saccades in people with PD, consistently noting that saccades are slower and smaller than those of control subjects. "Briand et al. reviewed a series of 15 studies of voluntary saccades and noted that all but one of these studies reported voluntary saccade performance inferior to that of control subjects in individuals with PD. Therefore, we hypothesize that saccadic eye movements performed during turns are also likely abnormal and may contribute to impaired turn performance. A disruption of the normal top-down rotation sequence by poor saccade timing or decreased saccade amplitude may contribute to the altered turning kinematics reported in those with PD. Hence, the purposes of this study were to determine whether saccadic eye movements during turning are impaired in individuals with PD and to determine if characteristics of the saccade that initiates a turn are predictive of ensuing turn performance.

\section{METHODS}

\section{Participants}

Twenty-three individuals with idiopathic PD and 19 age- and gender-matched controls participated in this investigation. Individuals with PD were recruited from a database of patients from Washington University School of Medicine's (WUSM) Movement Disorders Center. Control participants were recruited from the Volunteers for Health Database, posted flyers, and other healthy volunteer databases associated with WUSM. All subjects met the following inclusion criteria: aged 30 years or older, normal central (except for PD in the PD group) and peripheral neurological function, able to stand independently for at least 30 minutes and walk independently without an assistive device, no history of vestibular disease and no evidence or history of dementia. Exclusionary criteria included: any serious medical condition other than PD, use of neuroleptic or other dopamine-blocking drug, use of drug that might affect balance such as benzodiazepines, evidence of abnormality on brain imaging (previously done for clinical evaluations-not part of this research), history or evidence of other neurological deficit, such as previous stroke or muscle disease, and history or evidence of orthopedic, muscular, or psychological problem that may affect task performance during the study. Additionally, participants with PD were included based on a diagnosis of "definite PD" by a board certified neurologist, as previously described by Racette et al. (1999) based upon established criteria (Calne et al. 1992, Hughes et al. 1992) and were excluded if they had received surgical management of PD (e.g. pallidotomy or deep brain stimulation). All subjects gave informed consent to perform experimental procedures approved by the Human Research Protection Office at WUSM.

\section{Experimental procedures}

All study procedures were performed in the Locomotor Control Laboratory at WUSM. Participants with PD were tested OFF medication, i.e. after a 12-hour withdrawal of all anti- Parkinson medications. Before testing procedures commenced, the Movement Disorder Society Unified Parkinson's Disease Rating Scale (MDS-UPDRS) Motor Subscale III was administered according to Goetz et al. [31] by a trained rater. The MDS-UPDRS-III is a measure of severity of PD motor symptoms, as well as physical disability, and includes measures of rigidity, gait, tremor, hand/arm and leg 
movements (bradykinesia), speech, and facial expressions. The modified Hoehn and Yahr scale also was used to evaluate disease severity in PD [32].

During the experimental protocol, participants completed in-place turns of 90 degrees and 180 degrees amplitude. Instructions were given to perform the turns in a comfortable and normal fashion. No specific auditory or visual cues were provided to cue turn onset or completion other than directing subjects to "turn 90 degrees to face the wall beside you" or "turn 180 degrees to face the wall behind you", accordingly. Participants were instructed to begin the movement anytime after receiving the turn direction instruction of left or right for the given trial. Turns were completed to both the right and left in randomized order and all $90^{\circ}$ turns were completed prior to beginning the block of $180^{\circ}$ turns. Participants completed a minimum of 5 turns in each direction. Data quality was visually monitored in real time and additional turns were completed as needed to insure an adequate number of quality trials for analysis.

Full body kinematic data were captured using an eight camera, passive marker, 3-dimensional, highresolution motion capture system (Motion Analysis Corporation, Santa Rosa, CA) sampling at $100 \mathrm{~Hz}$ in Cortex software (Motion Analysis Corporation, Santa Rosa, CA). Thirty-eight retro-reflective markers were positioned on the head (top of head, back of head, left ear, right ear), trunk (left and right acromion, right scapula, sternal notch, xyphoid process, 7 th cervical vertebra, 12 th thoracic vertebra), pelvis (left and right anterior superior iliac spine, left and right posterior superior iliac spine, sacrum), both legs (greater trochanter, anterior thigh, medial and lateral femoral condyle, tibial tuberosity, front of shank, medial and lateral malleolus) and both feet (calcaneus, navicular, distal 2nd metatarsal). Ocuolmotor data were captured using a head-mounted infrared binocular eye tracking system (Applied Sciences Laboratory, Bedford, Ma) and electrooculography (EOG). Oculomotor data were captured synchronously at $1000 \mathrm{~Hz}$ on the same PC workstation with kinematic data in Cortex software.

\section{Data processing}

Individual kinematic marker data and analog data were filtered using 4 th order low-pass Butterworth filters. Marker data were filtered in Cortex with a cut-off frequency of $6 \mathrm{~Hz}$ while analog data were filtered in MotionMonitor (Innsport, Chicago, IL) with a cut-off frequency of $20 \mathrm{~Hz}$. Global and segment coordinate systems were defined in MotionMonitor with the posi- tive $\mathrm{X}$-axis pointing anteriorly, positive $\mathrm{Y}$-axis pointing to the left, and positive Z-axis pointing upward vertically. Rotations of the head, trunk, pelvis, and feet about global Z were extracted using a Z-X-Y Euler sequence. Subsequently, kinematic angle data and filtered analog data were exported for further processing in custom written MATLAB software (The Mathworks, Inc, Natick, MA).

Time of onset for segment rotations (relative to the global coordinate system) was determined by identifying the first frame at which the rotation reached five degrees above baseline. Similar criteria were used to identify turn offset, defined as the frame at which the rotation came within five degrees of maximal, final position. Eye tracker and EOG data were used to identify and measure saccades occurring just prior to and during turn performance. Saccades were identified visually and later confirmed to be true saccades if the maximum velocity of the eye movement exceeded 30 degrees/sec [33, 34] Onsets and offsets of the first saccade associated with each turn were identified visually. Using these time points, saccade amplitude, peak velocity, and timing of the first saccade relative to head and foot rotations were calculated. Example trials are shown for an individual with PD and a control in Fig. 1.

Individual trials were excluded from analysis if eye position or body segment rotations about the global Zaxis were not static for at least $1000 \mathrm{~ms}$ prior to turn onset. Trials were also excluded if artifacts in oculomotor data due to blinks, prolonged closure of eyelids, or other factors precluded measurement of the initial saccade. Remaining trials within a condition (90 or 180 degrees) were averaged to obtain a single data point for each subject. Left and right turns were combined for analysis as turn performance did not differ between leftward and rightward turns.

\section{Data analysis}

Independent Student's t-tests were used to compare between-group differences in turn performance and oculomotor performance during both 90 and 180 degree turns. Our primary variables of interest were the amplitude and velocity of the saccade initiating the turn, the total number of saccades performed during the turn, and the timing of the first saccade relative to onsets of head and foot rotations. The latencies between the first saccade and head/foot rotations were normalized to the duration of the first gait cycle and are reported as a percentage of the first gait cycle time. We also employed a linear regression model with turn duration as the dependent variable and number of sac- 

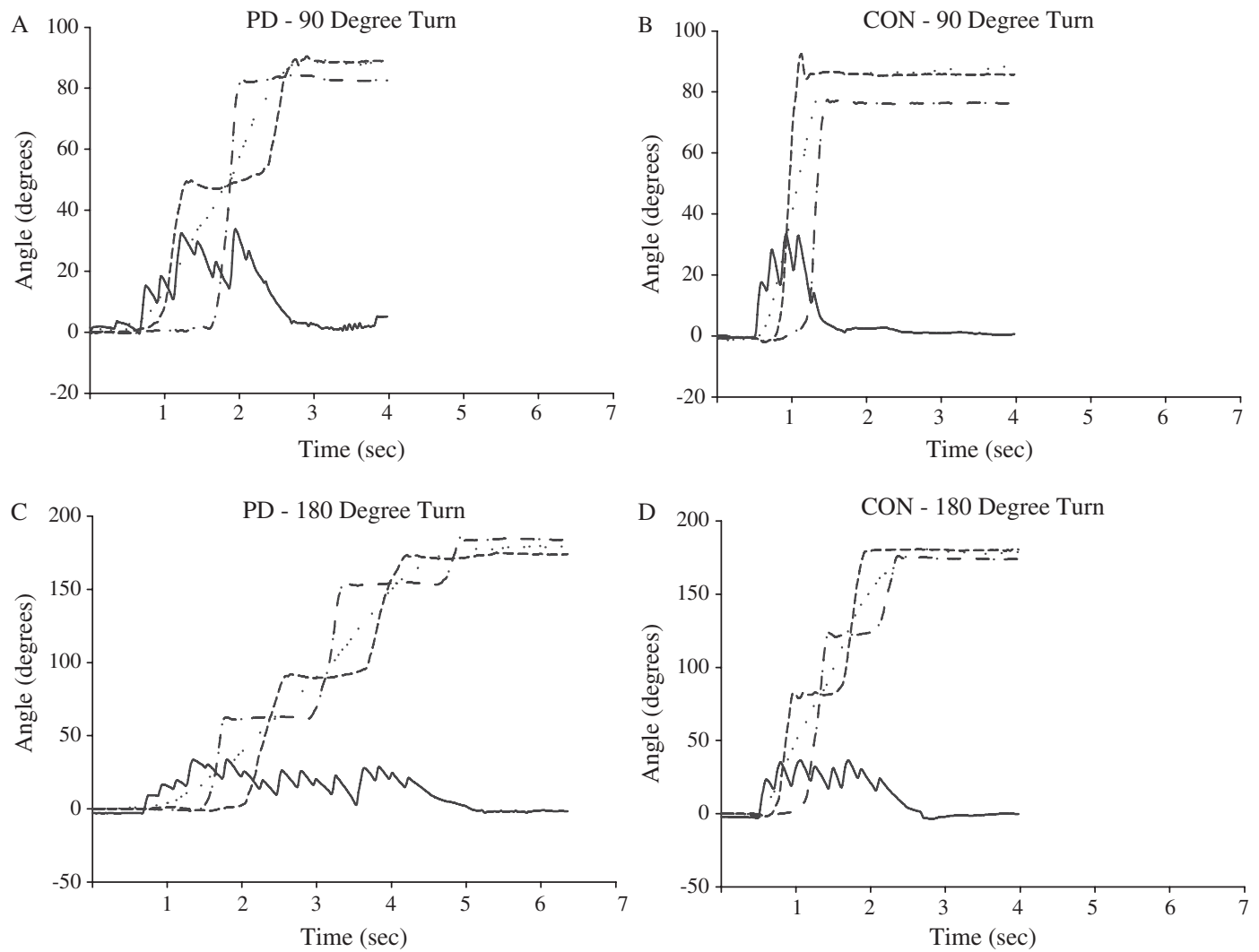

Fig. 1. Representative data from individual turn trials showing eye, head, and foot rotations in the horizontal plane. Panel A: Representative 90 degree turn performed by an individual with PD. The subject performed 8 saccades of varying amplitudes during the turn, and required 3 steps to complete the turn. Panel B: Representative 90 degree turn performed by a healthy control. The subject performed only 5 saccades during the turn and required only 2 steps and less time to complete the turn than the individual with PD. Panel C: Representative 180 degree turn performed by an individual with PD. The subject performed 15 saccades of varying amplitudes during the turn, and required 5 steps to complete the turn. Panel D: Representative 180 degree turn performed by a healthy control. The subject performed 8 saccades of more consistent amplitude than those performed by the individual with PD, and required only 4 steps and less time to complete the turn than the individual with PD.

cades, initial saccade velocity and normalized timing of the saccade relative to turn onset as the independent variables to identify the amount of variance in turn performance accounted for by characteristics the saccade initiating the turn. Saccade amplitude and the normalized timing of the saccade relative to head rotation onset were not included in the model as they were highly correlated with the included variables. The criterion for statistical significance was set at $p<0.05$.

\section{RESULTS}

Demographic data are displayed in Table 1. Data from three participants included in the 90 degree turn analysis could not be included in the analysis for the 180 degree turn due to poor oculomotor data quality. Conversely, one participant was included in the 180
Table 1

Subject demographics

\begin{tabular}{lccc}
\hline & $\begin{array}{c}\text { PD } \\
\left(90^{\circ} \text { turns }\right)\end{array}$ & $\begin{array}{c}\text { PD } \\
\left(180^{\circ} \text { turns }\right)\end{array}$ & Controls \\
\hline Age (years) & $68.7 \pm 10.2$ & $68.6 \pm 10.8$ & $68.8 \pm 11.4$ \\
Male/Female & $14 / 8$ & $13 / 7$ & $11 / 8$ \\
PD Characteristics & & & \\
Disease Duration (years) & $7.4 \pm 5.8$ & $6.8 \pm 5.6$ & \\
Hoehn \& Yahr Stage & $2.3 \pm 0.4$ & $2.3 \pm 0.4$ & \\
(\# in each stage) & Stage $1=1$ & Stage $1=1$ & \\
& Stage $2=9$ & Stage $2=7$ & \\
& Stage $2.5=10$ & Stage $2.5=10$ & \\
Freezing of Gait Score & Stage 3=2 & Stage 3=2 & \\
No. Freezers (FOG 3 $\geq 2)$ & $5.7 \pm 4.8$ & $5.8 \pm 5.0$ & \\
MDS-UPDRS III Score & $40.1 \pm 11.9$ & $38.7 \pm 11.5$ & \\
\hline
\end{tabular}

Values are means \pm standard deviations.

turn analysis but omitted from the 90 degree analysis for similar reasons. Regardless of turn type, age did not differ between PD and controls. 
Turn performance was impaired in PD compared with controls, with both 90 and 180 degree turns requiring more steps $(p<0.05)$ and a greater time to complete $(p<0.01)$. PD also performed a greater number of saccades during their turns, and the peak velocity of the initial saccade was slower in PD for both 90 and 180 degree turns $(p<0.01)$. The amplitude of the initial saccade was less in PD than in controls for 90 degree turns only $(p<0.01)$. The normalized latency between start of the first saccade and start of the first step (Norm E-F Index) was different between groups, with PD performing the first saccade earlier relative to the onset of foot rotation $(<0.05$, Table 2$)$.

The number of saccades, initial saccade amplitude, initial saccade velocity, and Norm E-F Index were all significantly correlated with turn duration (Fig. 2). Turn duration, which was highly correlated with the number of steps required to turn, was used as the dependent variable representing turn performance in our regression analysis. The linear regression model, which included both PD and controls, explained a significant amount of the variance in turn duration for both 90 degree $\left(R^{2}=0.481, \mathrm{~F}(3,27)=11.4, p<0.001\right)$ and 180 degree $\left(R^{2}=0.578, \mathrm{~F}(3,25)=16.0, p<0.001\right)$ turns. Table 3 reports the unstandardized (B) and standardized $(\beta)$ regression coefficients for these models.

Comparing freezers and non-freezers, turn duration and number of steps were greater in subjects who reported freezing of gait at least once per week on item 3 of the FOG questionnaire $(p<0.05)$. Mean values for initial saccade velocity and Norm E-F Index differed between freezers and non-freezers, but these comparisons did not reach statistical significance. Despite the lack of statistical significance, the effect sizes, measured using Cohen's $d$, were moderate to large. Effect size for saccade velocity between freezers and nonfreezers equaled 0.91 for 90 degree turns and 0.52 for 180 degree turns. Norm E-F Index effect sizes were 0.8 for 90 degrees turns and 0.86 for 180 degree turns. Number of saccades and initial saccade amplitude were similar between freezers and non-freezers. Data comparing freezers and non-freezers is presented in Table 4.

\section{DISCUSSION}

This study sought to determine whether saccadic eye movements performed during turning are impaired in individuals with PD and to determine if characteristics of the saccade that initiates a turn are predictive of ensuing turn performance. In confirmation of our hypotheses, saccadic eye movements were impaired during turning in persons with PD and these impairments were related to turning dysfunction. Individuals with PD used a greater number of saccades to complete both 90 and 180 degree turns, the initial saccade was both smaller (180 degrees only) and slower than that of controls, and the timing of the initial saccade relative to the turn onset was altered in those with PD. Furthermore, turn performance was impaired in persons with PD and approximately $50 \%$ of the variance in turn performance was explained by saccade performance across all participants. Differences in saccade performance between the 90 and 180 degree turns were largely predictable. The 180 degree turns required approximately twice as many saccades as the 90 degree turns and the amplitude of the initial saccade was similar between turn magnitudes for both groups. This suggests that the size of the turn-initiating saccade is constant for turns of 90 degrees and larger, and that simply more saccades are performed for large turns. Similarly, the delay between the first saccade and turn onset did not differ between the two turn magnitudes.

Previous research widely demonstrates that voluntary saccade performance is impaired in persons with PD [25-30]. These studies, however, have focused only on simple head-fixed tasks or on saccades performed

Table 2

Turn performance and oculomotor performance during 90 and 180 degree turns

\begin{tabular}{lccccccc}
\hline Measure & \multicolumn{3}{c}{$90^{\circ}$ Turns } & & \multicolumn{3}{c}{$180^{\circ}$ Turns } \\
\cline { 2 - 5 } \cline { 7 - 8 } & \multicolumn{2}{c}{ PD } & & Controls & & PD & Controls \\
\hline \# of Steps & $4.3 \pm 2.6$ & $*$ & $2.7 \pm 0.8$ & & $7.7 \pm 5.1$ & $*$ & $4.5 \pm 0.9$ \\
Turn Duration (seconds) & $2.1 \pm 0.8$ & $\dagger$ & $1.4 \pm 0.5$ & & $3.6 \pm 1.5$ & $\dagger$ & $2.4 \pm 0.7$ \\
\# of Saccades & $4.5 \pm 1.7$ & $\dagger$ & $3.1 \pm 1.4$ & & $8.9 \pm 3.2$ & $\dagger$ & $6.0 \pm 1.5$ \\
First Saccade Amplitude (degrees) & $20.6 \pm 8.1$ & & $25.7 \pm 8.4$ & & $17.4 \pm 4.6$ & $\dagger$ & $24.7 \pm 6.7$ \\
First Saccade Velocity (deg/sec) & $219.0 \pm 65.6$ & $\dagger$ & $273.1 \pm 41.1$ & & $206.7 \pm 61.2$ & $\dagger$ & $255.3 \pm 39.5$ \\
Norm E-H Index (\% of 1st gait cycle) & $19.4 \pm 19.3$ & & $11.5 \pm 6.1$ & & $26.8 \pm 25.0$ & $*$ & $13.4 \pm 7.2$ \\
Norm E-F Index (\% of 1st aait cvcle) & $45.4 \pm 33.9$ & $*$ & $25.4 \pm 9.7$ & & $52.3 \pm 38.1$ & $*$ & $28.1 \pm 11.5$ \\
\hline
\end{tabular}

Values are means \pm standard deviations.; $*$ Significantly different between groups, $p<0.05 ;{ }^{\dagger}$ Significantly different between groups, $p<0.01$ 
A

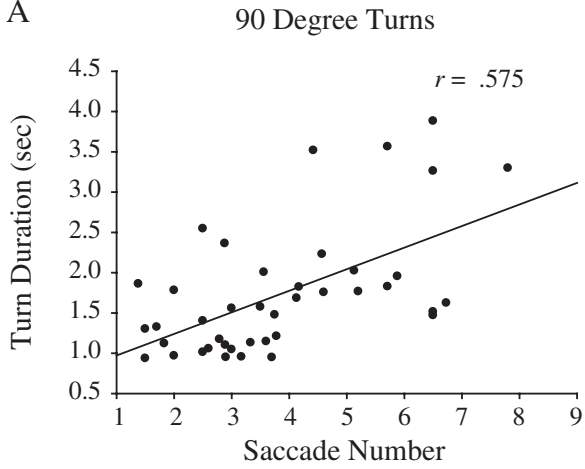

B

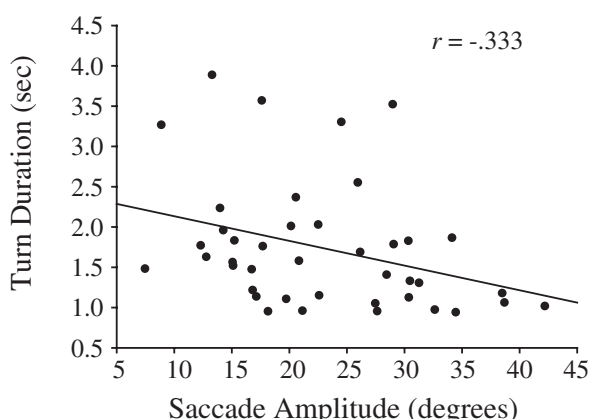

C

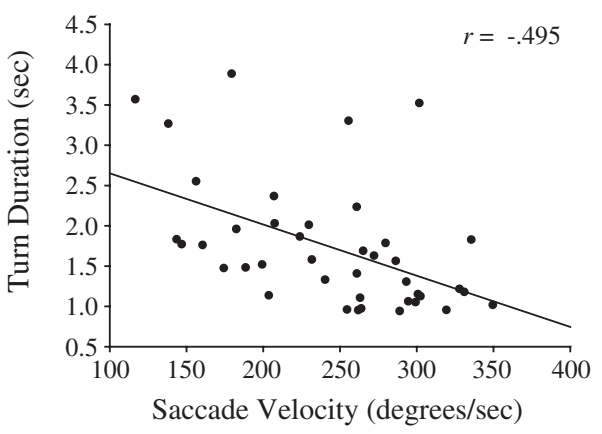

D

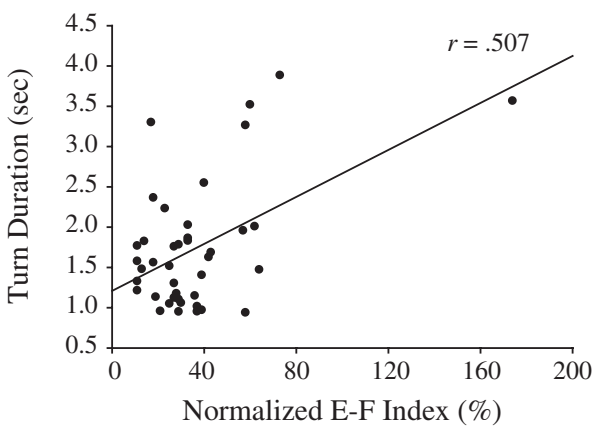

E

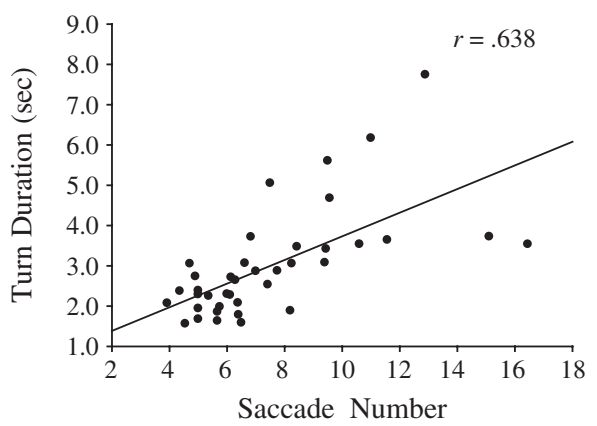

F

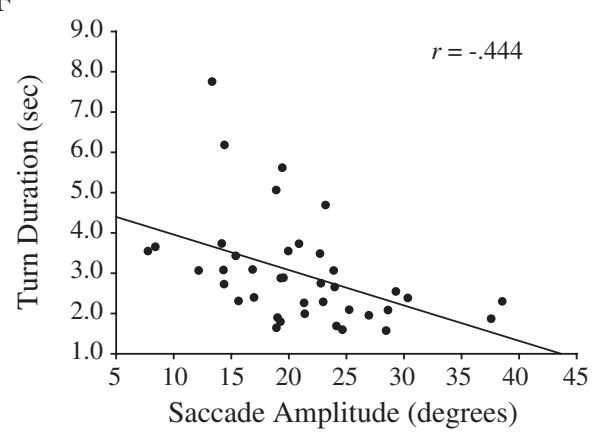

G

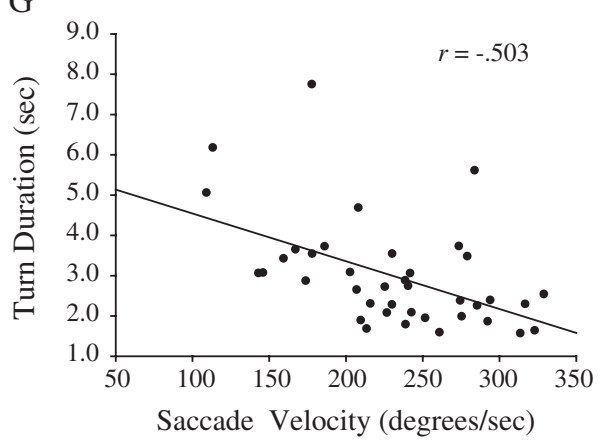

$\mathrm{H}$

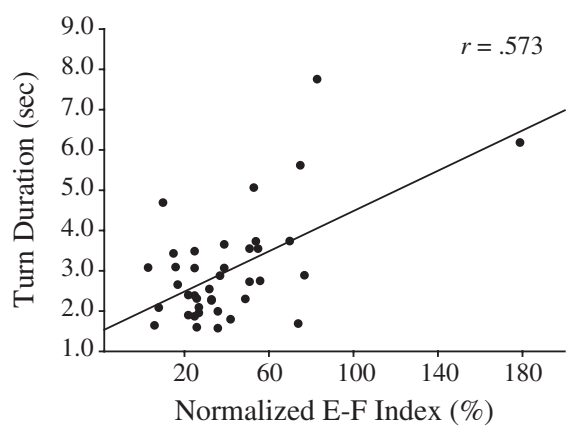

Fig. 2. Correlations between turn duration and various parameters of saccade performance. Correlations include all subjects from both the PD and control groups, with Pearson correlation coefficients shown in top right of each panel. The left column shows correlations of saccade number (A), amplitude of the first saccade (B), velocity of the first saccade (C), and normalized timing of the first saccade relative to the first step (D) for 90 degree turns. The right column (E-H) shows the same correlations for 180 degree turns. 
in conjunction with head movements from a seated position. Studying the oculomotor system using simple saccade paradigms has allowed researchers to better understand basal ganglia disorders using a simple, predictable, and well understood motor system. However, little information has been gathered from such studies regarding the implications of oculomotor impairments on functional activities in those with PD. To the best of our knowledge, this is the first study to report saccade performance during a more complex, functional task in people with PD. Our novel findings support previous work that voluntary saccades are impaired in PD and lend support to the idea that the eyes play a key role in turning. The turning sequence has been characterized in healthy controls and consists of a top-down rotation sequence led by the eyes and followed by rotations of the head, trunk, pelvis, and feet [20-23]. In individuals with PD this sequence is impaired, characterized by smaller intersegmental rotations and altered timing of segment rotations $[9,13,14]$. The present study reveals that the turning sequence in PD is also characterized by a longer than normal delay between the first saccade and the initiation of the gait cycle, as well as a smaller and slower saccade at the beginning of the turn. Functionally, this manifests in reduced turn performance. As evidenced by the strong correlations between saccade performance (the number of saccades, saccade velocity, and saccade timing) and turn performance (number of steps and turn duration), the degree of oculomotor impairment may impact turn quality.

Our finding of a greater delay between the initial saccade and the rest of the turning sequence in the PD group is contrary to our hypothesis. Expanding the PD en-bloc turning phenomenon to include eye movements, one would expect the eyes to rotate more in sync with the head, trunk and feet, as opposed to our observation of a longer latency between the eyes and feet. Our PD group actually performed the first saccade much earlier in the rotation sequence than did
Table 3

Results of linear regression analysis

\begin{tabular}{|c|c|c|c|c|c|}
\hline & & B & $\mathrm{SE}(\mathrm{B})$ & $\beta$ & $\mathrm{p}$ \\
\hline \multirow[t]{3}{*}{$90^{\circ}$ Turns } & \# Saccades & 18.24 & 6.40 & 0.392 & 0.0007 \\
\hline & Saccade Velocity & -0.232 & 0.18 & -0.180 & 0.211 \\
\hline & Norm E-F Index & 94.59 & 36.93 & 0.329 & 0.015 \\
\hline \multirow[t]{3}{*}{$180^{\circ}$ Turns } & \# Saccades & 18.72 & 5.79 & 0.407 & 0.003 \\
\hline & Saccade Velocity & -0.283 & 0.28 & -0.248 & 0.048 \\
\hline & Norm E-F Index & 147.01 & 53.01 & 0.337 & 0.009 \\
\hline
\end{tabular}

$90^{\circ}$ Turns, $R^{2}=.481180^{\circ}$ Turns, $R^{2}=.578$

the controls, and the longer latencies were unexpectedly associated with a longer turn duration and more steps. This finding may be explained by a generalized bradykinesia that affects both the motor and oculomotor systems. While the basal ganglia are often described as having distinct loops for oculomotor and motor control, recent evidence suggests an overlap in control of both eye and limb movements by the subthalamic nucleus (STN), as neurons in the STN respond to both voluntary saccades and limb movements [35]. Therefore, the greater delay between eye movement and turn onset seen in PD may be the result of a dysfunctional common motor pathway responsible for an overall bradykinetic turn sequence. Based on this, deep brain stimulation (DBS) may prove beneficial for improving turn performance in PD by enhancing both eye and limb movements. Levodopa therapy, the most common treatment for those with $\mathrm{PD}$, provides minimal improvement in both turn performance and voluntary saccade performance $[36,37]$. However, DBS of the STN in persons with PD has shown considerable efficacy in improving motor performance, including gait and performance of voluntary and reflexive saccades [38-40]. However, no studies to date have examined the effect of DBS on turn performance, nor the effect of DBS on saccade function during functional tasks. Therefore, future work should target the effects of STN-DBS on turn performance and associated oculomotor performance.

Table 4

Comparison of freezers and non-freezers

\begin{tabular}{|c|c|c|c|c|c|c|}
\hline & \multicolumn{3}{|c|}{$90^{\circ}$ Turns } & \multicolumn{3}{|c|}{$180^{\circ}$ Turns } \\
\hline & Freezers $(n=8)$ & & Non-Freezers $(n=14)$ & Freezers $(n=8)$ & & Non-Freezers $(n=12)$ \\
\hline Disease Duration & $8.6 \pm 7.0$ & & $6.7 \pm 5.2$ & $8.3 \pm 6.7$ & & $5.8 \pm 4.8$ \\
\hline MDS-UPDRS III Score & $40.1 \pm 13.1$ & & $40.1 \pm 11.7$ & $39.9 \pm 12.9$ & & $37.8 \pm 11.0$ \\
\hline \# Saccades & $4.5 \pm 2.0$ & & $4.6 \pm 1.6$ & $9.1 \pm 2.6$ & & $8.8 \pm 3.7$ \\
\hline Saccade Amplitude (degrees) & $20.6 \pm 8.5$ & & $20.7 \pm 8.2$ & $18.2 \pm 3.4$ & & $16.9 \pm 5.4$ \\
\hline Saccade Velocity (deg/sec) & $183.8 \pm 59.8$ & & $239.2 \pm 61.7$ & $187.7 \pm 61.5$ & & $219.4 \pm 60.2$ \\
\hline Norm E-F Index & $61.1 \pm 49.0$ & & $36.4 \pm 18.2$ & $70.8 \pm 48.1$ & & $40.0 \pm 24.8$ \\
\hline Total Steps & $6.4 \pm 3.6$ & $*$ & $3.1 \pm 0.5$ & $11.1 \pm 6.7$ & $*$ & $5.4 \pm 1.1$ \\
\hline Turn Duration (seconds) & $2.8 \pm 8.1$ & $\dagger$ & $1.6 \pm 0.4$ & $4.7 \pm 1.7$ & $*$ & $2.8 \pm .77$ \\
\hline
\end{tabular}

Values are means \pm standard deviations.; *Significantly different between groups, $p<0.05 ;{ }^{\dagger}$ Significantly different between groups, $p<0.05$ 
Studies extending beyond PD corroborate a relationship between oculomotor dysfunction and gait impairments; a relationship that appears to be related to risk of falling in a range of populations. In a study comparing elderly individuals who were at high risk for falling with those at low risk for falling, a longer delay between horizontal saccade initiation and initiation of footlift was observed in the high-risk group during a precise walking task [41]. Differences in gaze behavior have also been shown between adult fallers and non-fallers [18]. In patients with progressive supranuclear palsy (PSP), those with more severe gaze palsy displayed an altered stepping pattern when navigating obstacles, placing them at higher risk for trips and falls [42]. In our study, subjects who reported FOG at least once per week displayed turn performance deficits and altered saccade timing and velocity, although the comparison of oculomotor measures failed to reach statistical significance, possibly due to the small group sizes. Disease severity (MDSUPDRS III) and duration were not different between freezers and non-freezers, illustrating that FOG is a specific pathology not present in all PD patients regardless of disease stage or severity [2]. While we did not obtain fall history records in this study, FOG has been shown to be a risk factor for falling, and thus the freezers in our study likely represent a sample of patients at higher risk for falls and fall-related injuries. Taken together, our study and those of other pathological populations suggest a relationship between fall risk and gait/oculomotor function. Therefore, rehabilitation strategies aimed at decreasing the risk of falls during ambulation, and in particular during turning, are important.

Cueing has received considerable attention over the past decade as a means of improving temporal and spatial parameters of gait in persons with PD. Rhythmic auditory, visual, and attentional cues have been shown to improve stride length and gait velocity during straight walking [43-46]. However, the ability of cues to improve turning performance is less well understood. When rhythmic auditory cues were used during a U-turn task, only step time variability was improved among a number of turn performance parameters [16]. In contrast, another study found that rhythmic auditory and somatosensory cues improved turn time in a functional task (carrying a tray) [47]. Clearly, more work is necessary to determine the effect of cues on turning, and based on the importance of oculomotor function during turning, using cues to promote a more appropriate oculomotor strategy during turns should be of interest.

\section{Limitations}

One limitation of this study is that saccades were measured using two separate measurement systems. The infrared binocular eye tracking system served as our primary measurement tool, with EOG serving a secondary role. Due to the technical nature of measuring pupil and corneal reflections using the infrared system, quality infrared data could not be obtained from all participants. In such cases, EOG data were used for analysis. To verify agreement between these two measurement systems, infrared and EOG data were compared using data from participants for whom we had both data sets. When comparing the timing, amplitude, and velocity of the initial saccade, values obtained from the two systems compared exceptionally well. Therefore, the authors felt confident in pooling data obtained from either measurement system. Another limitation of this study is that measurement occurred in a laboratory setting and thus participants were aware that their performance was being monitored. Hence, it is possible that participants' oculomotor and turning performance may have differed from their usual performance in a more natural setting. The authors think, however, that such effects are minimal and would have been experienced similarly by both groups, thus not detracting for our findings.

\section{Conclusions and future directions}

It is evident that turning difficulty is a primary trigger for freezing and falls in PD, and our study indicates that impaired voluntary saccades may contribute significantly to this problem. Rehabilitative strategies might consider focusing on cueing persons with PD to initiate turns with a more appropriate top-down rotation sequence, initiated by a large amplitude saccade prior to commencing the gait cycle. Accordingly, future research should be directed towards studying the effects of cueing and practice on the ability to improve saccade performance during turns, and whether such improvements offer meaningful improvements in turn performance and related fall risk. Additionally, future work may assess the effects of therapeutic interventions (e.g. deep brain stimulation) on such variables.

\section{ACKNOWLEDGMENTS}

We thank Marie McNeely for assistance with data collection, Brian Morrell for assistance with data processing, and Dr. Richard Abrams for assistance with project design and pilot testing. This publication was 
made possible by R01 HD056015, the Barnes Jewish Hospital Foundation, and Grant Number UL1 RR024992 from the National Center for Research Resources (NCRR), a component of the National Institutes of Health (NUT), and NUT Roadmap for Medical Research. Its contents are solely the responsibility of the authors and do not necessarily represent the official view of NCRR or NUT. Additional support came from the St. Louis Chapter of the American Parkinson Disease Association (APDA) and the APDA Advanced Center for PD Research at Washington University in St. Louis.

\section{REFERENCES}

[1] Giladi N, McMahon D, Przedborski S. et al. (1992) Motor blocks in Parkinson's disease. Neurology, 42(2), 333-339.

[2] Bloem BR, Hausdorff JM, Visser JE, \& Giladi N (2004) Falls and freezing of gait in Parkinson's disease: a review of two interconnected, episodic phenomena. Mov Disord, 19(8), 871884.

[3] Bloem BR, Grimbergen YA, Cramer M, Willemsen M, \& Zwinderman AH (2001) Prospective assessment of falls in Parkinson's disease. J Neurol, 248(11), 950-958.

[4] Cumming RG \& Klineberg RJ (1994) Fall frequency and characteristics and the risk of hip fractures. $J$ Am Geriatr Soc, 42(7), 774-778.

[5] Melton LJ, 3Rd, Leibson CL, Achenbach SJ, et al. (2006) Fracture risk after the diagnosis of Parkinson's disease: influence of concomitant dementia. Mov Disord, 21(9), 1361-1367.

[6] Bacon WE (1996) Secular trends in hip fracture occurrence and survival: age and sex differences. J Aging Health, 8(4), 538-553.

[7] Stack E \& Ashburn A (2008) Dysfunctional turning in Parkinson's disease. Disabil Rehabil, 30(16), 1222-1229.

[8] Morris ME, Huxham F, McGinley J, Dodd K, \& Iansek R (2001) The biomechanics and motor control of gait in Parkinson disease. Clin Biomech (Bristol, Avon), 16(6), 459-470.

[9] Hong M, Perlmutter JS, \& Earhart GM (2009) A kinematic and electromyographic analysis of turning in people with Parkinson disease. Neurorehabil Neural Repair, 23(2), 166176.

[10] Gruendlinger L, Yogev G, Springer S, \& Hausdorff JM (2005) Does turning differ from walking? Turning duration, gait indices and fall risk in Parkinson's disease and idiopathic fallers. Gait and Posture, 21(S1):S94.

[11] Mak MKY, Chan CWY, \& Palta AE (2005) Turning strategy in patients with Parkinson's disease. Gait and Posture, 21(S1):S95

[12] Stack EL, Ashburn AM, \& Jupp KE (2006) Strategies used by people with Parkinson's disease who report difficulty turning. Parkinsonism Relat Disord, 12(2), 87-92.

[13] Crenna P, Carpinella I, Rabuffetti M et al. (2007) The association between impaired turning and normal straight walking in Parkinson's disease. Gait Posture, 26(2), 172-178.

[14] Visser JE, Voermans NC, Oude Nijhuis LB et al. (2007) Quantification of trunk rotations during turning and walking in Parkinson's disease. Clin Neurophysiol, 118(7), 16021606.
[15] Huxham F, Baker R, Morris ME, \& Iansek R (2008) Head and trunk rotation during walking turns in Parkinson's disease. Mov Disord, 23, 10 1391-1397.

[16] Willems AM, Nieuwboer A, Chavret F et al. (2007) Turning in Parkinson's disease patients and controls: the effect of auditory cues. Mov Disord, 22(13), 1871-1878.

[17] Mak MK, Patla A, \& Hui-Chan C (2008) Sudden turn during walking is impaired in people with Parkinson's disease. Exp Brain Res, 190(1), 43-51.

[18] Chapman GJ \& Hollands MA (2006) Evidence for a link between changes to gaze behaviour and risk of falling in older adults during adaptive locomotion. Gait Posture, 24(3), 288-294.

[19] Crowdy KA, Kaur-Mann D, Cooper HL, Mansfield AG, Offord JL, \& Marple-Horvat DE (2002) Rehearsal by eye movement improves visuomotor performance in cerebellar patients. Exp Brain Res, 146(2), 244-247.

[20] Grasso R, Prevost P, Ivanenko YP, \& Berthoz A (1998) Eye-head coordination for the steering of locomotion in humans: an anticipatory synergy. Neurosci Lett, 253(2), 115118.

[21] Imai T, Moore ST, Raphan T, \& Cohen B (2001) Interaction of the body, head, and eyes during walking and turning. Exp Brain Res, 136(1), 1-18.

[22] Hollands MA, Patla AE, \& Vickers JN (2002) "Look Where You're Going!": gaze behaviour associated with maintaining and changing the direction of locomotion. Exp Brain Res, 143(2), 221-230.

[23] Hollands MA, Ziavra NV, \& Bronstein AM (2004) A new paradigm to investigate the roles of head and eye movements in the coordination of whole-body movements. Exp Brain Res, 154(2), 261-266.

[24] Vallis LA, Patla AE, \& Adkin AL (2001) Control of steering in the presence of unexpected head yaw movements. influence on sequencing of subtasks. Exp Brain Res, 138(1), 128-134.

[25] DeJong JD \& Jones GM (1971) Akinesia, hypokinesia, and bradykinesia in the oculomotor system of patients with Parkinson's disease. Exp Neurol, 32(1), 58-68.

[26] Lueck CJ, Tanyeri S, Crawford TJ, Henderson L, \& Kennard C (1990) Antisaccades and remembered saccades in Parkinson's disease. J Neurol Neurosurg Psychiatry, 53(4), 284-288.

[27] Ventre J, Zee DS, Papageorgiou H, \& Reich S (1992) Abnormalities of predictive saccades in hemi-Parkinson's disease. Brain, 115, 1147-1165.

[28] Crawford T, Goodrich S, Henderson L, \& Kennard C (1989) Predictive responses in Parkinson's disease: manual keypresses and saccadic eye movements to regular stimulus events. J Neurol Neurosurg Psychiatry, 52(9), 1033-1042.

[29] Briand KA, Strallow D, Hening W, Poizner H, \& Sereno AB (1999) Control of voluntary and reflexive saccades in Parkinson's disease. Exp Brain Res, 129(1), 38-48.

[30] MacAskill MR, Anderson TJ, \& Jones RD (2002) Adaptive modification of saccade amplitude in Parkinson's disease. Brain, 125, 1570-1582.

[31] Goetz CG, Tilley BC, Shaftman SR et al. (15) Movement disorder society-sponsored revision of the unified Parkinson's disease rating scale (MDS-UPDRS): scale presentation and clinimetric testing results. Mov Disord, 23(15), 2129-2170.

[32] Goetz CG, Poewe W, Rascol O et al. (2004) Movement disorder society task force report on the Hoehn and Yahr staging scale: status and recommendations. Mov Disord, 19(9), 10201028

[33] Chan F, Armstrong IT, Pari G, Riopelle RJ, \& Munoz DP (2005) Deficits in saccadic eyemovement control in Parkinson's disease. Neuropsychologia, 43(5), 784-796. 
[34] Versino M, Zavanone C, Colnaghi S et al. (2005) Binocular control of saccades in idiopathic Parkinson's disease. Ann $N$ YAcadSci, 1039, 588-592.

[35] Fawcett AP, Dostrovsky JO, Lozano AM, \& Hutchison WD (2005) Eye movement-related responses of neurons in human subthalamic nucleus. Exp Brain Res, 162(3), 357-365.

[36] Vermersch AI, Rivaud S, Vidailhet M et al. (1994) Sequences of memory-guided saccades in Parkinson's disease. Ann Neurol, 35(4), 487-490.

[37] Hong M \& Earhart GM (2010) Effects of medication on turning deficits in individuals with Parkinson's disease. J Neurol Phys Ther, 34(1), 11-16.

[38] Rivaud-Pechoux S, Vermersch AI, Gaymard B et al. (2000) Improvement of memory guided saccades in Parkinsonian patients by high frequency subthalamic nucleus stimulation. J Neurol Neurosurg Psychiatry, 68(3), 381-384.

[39] Sauleau P, Pollak P, Krack P et al. (2008) Subthalamic stimulation improves orienting gaze movements in Parkinson's disease. Clin Neurophysiol, 119(8), 1857-1863.

[40] Yugeta A, Terao Y, Fukuda H et al. (2010) Effects of STN stimulation on the initiation and inhibition of saccade in Parkinson disease. Neurology, 74(9), 743-748.

[41] Greany JF \& Di Fabio RP (2008) Saccade to stepping delays in elders at high risk for falling. Aging Clin Exp Res, 20(5), 428-433.
[42] Di Fabio RP, Zampieri C, \& Tuite P (2008) Gaze control and foot kinematics during stair climbing: characteristics leading to fall risk in progressive supranuclear palsy. Phys Ther, $\mathbf{8 8}(2)$, 240-250.

[43] Lohnes CA \& Earhart GM (2011) The impact of attentional, auditory, and combined cues on walking during single and cognitive dual tasks in Parkinson disease. Gait Posture, 33(3), 478-483.

[44] Mclntosh GC, Brown SH, Rice RR, \& Thaut MH (1997) Rhythmic auditory-motor facilitation of gait patterns in patients with Parkinson's disease. J Neurol Neurosurg Psychiatry, 62(1), 22-26.

[45] Morris ME, Iansek R, Matyas TA, \& Summers JJ (1996) Stride length regulation in Parkinson's disease. Normalization strategies and underlying mechanisms. Brain, 119, 551-568.

[46] Nieuwboer A, Kwakkel G, Rochester L et al. (2007) Cueing training in the home improves gaitrelated mobility in Parkinson's disease: the rescue trial. J Neurol Neurosurg Psychiatry, 78(2), 134-140.

[47] Nieuwboer A, Baker K, Willems AM et al. (2009) The shortterm effects of different cueing modalities on turn speed in people with Parkinson's disease. Neurorehabil Neural Repair, 23(8), 831-836. 